\title{
A novel polycaprolactone/carbon nanofiber composite as a conductive neural guidance channel: an in vitro and in vivo study
}

\author{
Saeed Farzamfar ${ }^{1} \cdot$ Majid Salehi ${ }^{2,3} \cdot$ Seyed Mohammad Tavangar ${ }^{4} \cdot J_{a v a d ~ V e r d i}{ }^{1} \cdot K$ Korosh Mansouri ${ }^{5} \cdot$ Arman $\mathbf{A i}^{6}$. \\ Ziba Veisi Malekshahi ${ }^{1} \cdot$ Jafar $\mathbf{A i}^{1}$
}

Received: 30 July 2019 / Accepted: 16 September 2019 / Published online: 12 December 2019

(C) The Author(s) 2019

\begin{abstract}
The current study aimed to investigate the potential of carbon nanofibers to promote peripheral nerve regeneration. The carbon nanofiber-imbedded scaffolds were produced from polycaprolactone and carbon nanofibers using thermally induced phase separation method. Electrospinning technique was utilized to fabricate polycaprolactone/collagen nanofibrous sheets. The incorporation of carbon nanofibers into polycaprolactone's matrix significantly reduced its electrical resistance from $4.3 \times 10^{9} \pm 0.34 \times 10^{9} \Omega$ to $8.7 \times 10^{4} \pm 1.2 \times 10^{4} \Omega$. Further in vitro studies showed that polycaprolactone/carbon nanofiber scaffolds had the porosity of $82.9 \pm 3.7 \%$ and degradation rate of $1.84 \pm 0.37 \%$ after 30 days and $3.58 \pm 0.39 \%$ after 60 days. The fabricated scaffolds were favorable for PC-12 cells attachment and proliferation. Neural guidance channels were produced from the polycaprolactone/carbon nanofiber composites using water jet cutter machine then incorporated with PCL/ collagen nanofibrous sheets. The composites were implanted into severed rat sciatic nerve. After 12 weeks, the results of histopathological examinations and functional analysis proved that conductive conduit out-performed the non-conductive type and induced no toxicity or immunogenic reactions, suggesting its potential applicability to treat peripheral nerve damage in the clinic.
\end{abstract}

Keywords Carbon nanofiber $\cdot$ Polycaprolactone $\cdot$ Electrical conductivity $\cdot$ Sciatic nerve regeneration

\section{Introduction}

Unlike the central nervous system, peripheral nerves have the capacity to regenerate the injured tissue (Faroni et al. 2015). However, in most cases this capacity is disrupted

Jafar Ai

jafar_ay@tums.ac.ir

1 Department of Tissue Engineering and Applied Cell Sciences, School of Advanced Technologies in Medicine, Tehran University of Medical Sciences, Tehran, Iran

2 Department of Tissue Engineering, School of Medicine, Shahroud University of Medical Sciences, Shahroud, Iran

3 Tissue Engineering and Stem Cells Research Center, Shahroud University of Medical Sciences, Shahroud, Iran

4 Department of Pathology, Shariati Hospital, Tehran University of Medical Sciences, Tehran, Iran

5 Neuromusculoskletal Research Centre Firozgar Hospital, Iran University of Medical Sciences, Tehran, Iran

6 School of Medicine, Tehran University of Medical Sciences, Tehran 141556447, Iran and peripheral nerve repair is incomplete with poor functional recovery ( $\mathrm{Gu}$ et al. 2014). Currently, the treatment of choice is end-to-end epineurial suturing of the proximal and distal nerve stumps (Siemionow and Brzezicki 2009). In case of critical size defects, where tension-free neurorrhaphy is not possible, autologous nerve grafting is considered as the gold standard (Muheremu and Ao 2015). However, this treatment modality is associated with several shortcomings including the sacrifice of a healthy nerve, need for secondary surgery, and extra damage to donor site (Habre et al. 2018). Collectively, these disadvantages have driven efforts toward developing alternative therapeutic strategies. With the progress of tissue engineering, a variety of natural and/or synthetic polymers have been utilized to produce neural guidance channels (NGCs) in an attempt to substitute autologous nerve grafts (Farzamfar et al. 2018; Nectow et al. 2011). The NGCs preclude the invasion of fibrous tissue into the gap between two stumps. In addition, NGCs can entrap and enrich the neurotrophic factors within the channel and build a permissive environment for nerve regeneration (Muheremu and Ao 2015). 
Appropriate choice of biomaterial and fabrication method is crucial to design an ideal NGC (Sarker et al. 2018). In this regard, a variety of synthetic and/or natural materials have been tested in neural tissue engineering (Nectow et al. 2011; Yu et al. 2011). Among synthetic biomaterials poly( $\varepsilon$-caprolactone) (PCL) due to its biocompatibility, bioresorbability, and lack of immunogenicity provide a favorable environment in the context of tissue repair and reconstruction (Smith et al. 2015). However, cell affinity towards PCL is generally poor as a result of its lack of cell attachment sites and high hydrophobicity (Lee et al. 2012; Schnell et al. 2007). To improve cell-scaffold interactions, one useful strategy is to incorporate cell-recognition domains such as Arg-Gly-Asp (RGD) groups and extracellular matrix (ECM) bioactive proteins into the PCL scaffolds (Zheng et al. 2012). Collagen is the main component of native ECM. Therefore, the selection of collagen as a scaffolding material is most favored in tissue engineering. However, fast degradation may hamper its application as a platform to guide axonal growth (Glowacki and Mizuno 2008). Therefore, we used a combination of PCL and collagen to develop NGCs. Developing scaffolds with structural similarity to natural ECM at the nanoscale has a significant effect on organization of cells and the corresponding tissue properties (Lannutti et al. 2007). Furthermore, the optimum porosity of the NGCs wall will facilitate metabolites diffusion which is one of the essential requirements of an NGC (Keane and Badylak 2014). To provide such scaffolding structures, the combination of thermally induced phase separation (TIPS) and electrospinning methods can be used. Electrospun fibers have been investigated as promising tissue engineering scaffolds for peripheral nerve regeneration (Prabhakaran et al. 2008; Wang et al. 2008). TIPS method can be utilized to yield porous scaffolds with suitable interconnectivity of the pores (Dong 2015; Salehi et al. 2015). It has been shown that electrically conductive NGCs have superior regenerative capacity than non-conductive NGCs (Salehi et al. 2018). Since PCL and collagen are non-conductive, electrical properties of PCL/collagen composite scaffold can be improved by incorporation of filler materials. In this regard, it has been previously shown that incorporation of carbon nanofibers (CNF) in the scaffolds can endow them with the conduction capability (Stout et al. 2011). Since the effects of CNFs in peripheral nerve regeneration have not been studied yet, the basic aim of the current research was to investigate the regenerative potential of PCL/CNF conduits in a rat model of sciatic nerve defect.

\section{Methods and materials}

The polymers and solvents were obtained from Sigma-Aldrich (St. Louis, USA) and Merck (Darmstadt, Germany), respectively, unless otherwise noted.

\section{Fabrication of the NGCs through thermally induced phase separation and electrospinning methods}

Carbon nanofibers (US Research Nanomaterials Inc. Cas no. 308068-56-6) were dispersed in 1,4-dioxane using a magnetic stirrer for $6 \mathrm{~h}$ and then ultra-sonicated for $3 \mathrm{~h}$ at room temperature. After this time period, polycaprolactone (PCL, $\left.M_{\mathrm{w}} \sim 80.000 \mathrm{kDa}\right)$ was added to the $\mathrm{CNF}$ containing 1,4-dioxane to the final concentration of $6 \% \mathrm{w} / \mathrm{v}$ (CNF weight in the solution was $10 \%$ of PCL weight) and stirred for $24 \mathrm{~h}$ and then ultra-sonicated to homogenously disperse the CNF in the PCL matrix. The resulting mixture was transferred to $-20{ }^{\circ} \mathrm{C}$ and maintained for $4 \mathrm{~h}$. After $4 \mathrm{~h}$, the solidified mixture was transferred to $-80^{\circ} \mathrm{C}$ and kept overnight. The sample was freezedried (Telstar co., Terrassa, Spain) for $48 \mathrm{~h}$. The fabricated scaffold was shaped into 14-mm conduits using a water jet cutter machine. In order to fabricate PCL/collagen nanofibers, PCL and collagen were separately dissolved in acetic acid at concentrations of $14 \%(\mathrm{w} / \mathrm{v})$ and $5 \%(\mathrm{w} / \mathrm{v})$, respectively, and then mixed at the volume ratio of 70:30. The prepared solution was transferred into a 10-mL disposable syringe and fixed in a syringe pump (SP1000, Fanavaran Nano-Meghyas, Iran). An 18 gauge stainless metal needle was placed at the tip of the syringe and the needle tip to collecting mandrel distance was set at $15 \mathrm{~cm}$. Electrospinning was performed by applying a positive high voltage $(18 \mathrm{kV})$ and the polymer was fed at the rate of $0.5 \mathrm{~mL} / \mathrm{h}$. Electrospun matrices were cut into thin thread like pieces and put into the NGC's lumen at the time of implantation. Schematic illustration of the NGC's fabrication process is shown in Fig. 1.

\section{Characterization of the scaffolds}

\section{Scanning electron microscopy (SEM) analysis of scaffolds}

The microstructure of the produced composite was evaluated by scanning electron microscope (SEM; DSM 960A, Zeiss, Germany) followed by coating with gold for $250 \mathrm{~s}$ using a sputter coater (SCD 004, Balzers, Germany).

\section{Weight loss measurement}

In vitro degradation measurement was carried out by immersing the NGCs in $10 \mathrm{~mL}$ of $\mathrm{PBS}$ at $37{ }^{\circ} \mathrm{C}$ in an incubator. After selected times (30 and 60 days), conduits were 


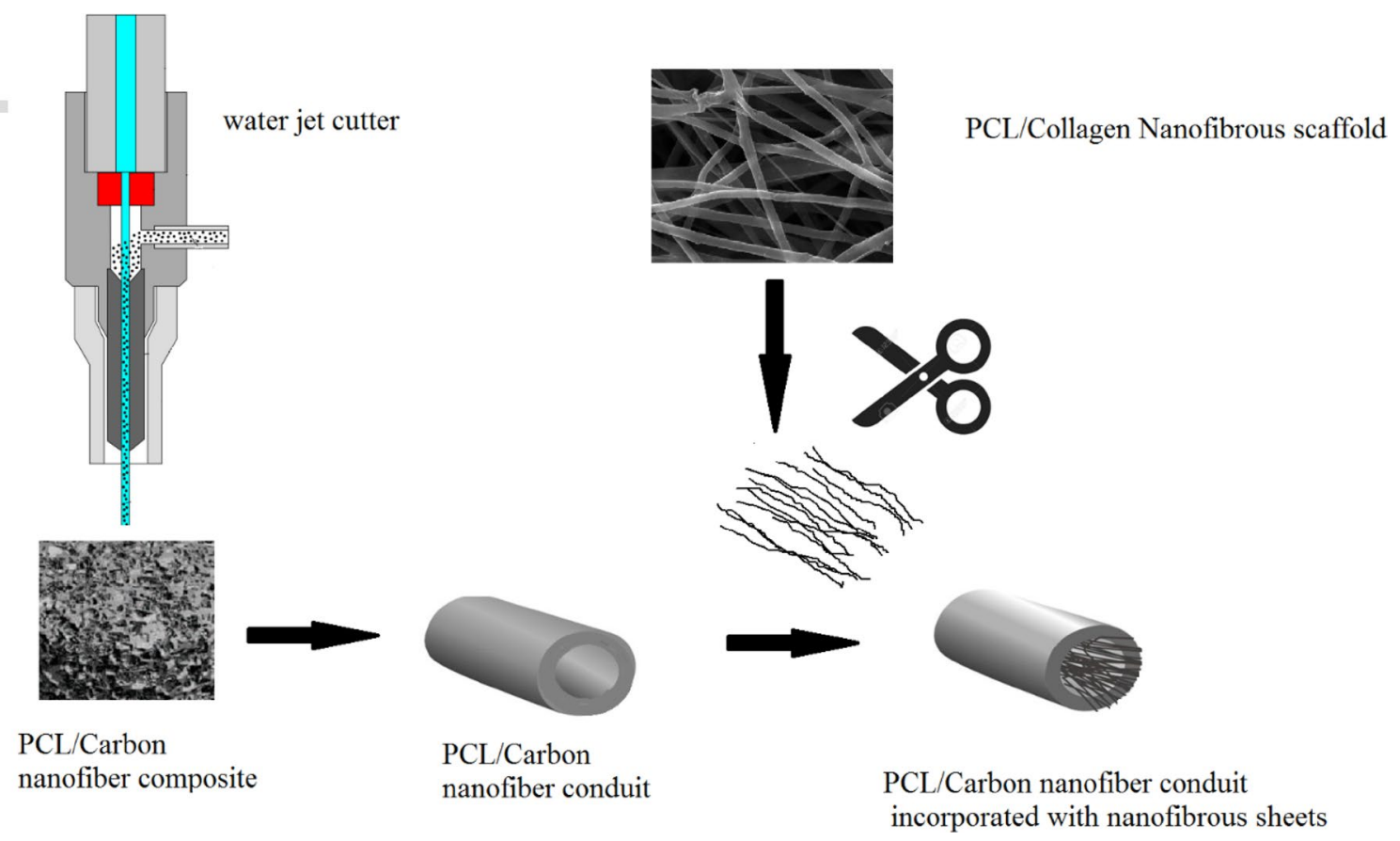

Fig. 1 Schematic illustration for the production of the neural guidance channels

taken out and freeze-dried. Weight loss was calculated using the following equation:

Weight loss $(\%)=\frac{W_{0}-W_{1}}{W_{0}} \times 100$,

where " $W_{0}$ " indicates the initial weight of NGCs and " $W_{1}$ " is the dry weight of the NGCs after removing from the PBS solution.

\section{Porosity measurement}

Liquid displacement method and the flowing equation were used to determine the NGC's porosity:

Porosity $(\%)=\frac{V 1-V 3}{V 2-V 3} \times 100$,

where $V_{1}$ stands for the volume of $96 \%$ ethanol before the immersion of NGC, $V_{2}$ indicates its volume after NGC immersion and $V_{3}$ is the volume of the ethanol after the NGC removal.

\section{Conductivity measurement}

The surface resistance of fabricated scaffolds was evaluated using a conventional four-probe method. The composite scaffolds were cut in $2 \times 3 \mathrm{~cm}^{2}$ shape and put on the fourprobe instrument (Santa Nama Javan Co). By applying a voltage the corresponding electrical current was recorded.
The surface resistance of the samples was determined by using the following equation:

$R_{\mathrm{s}}(\Omega)=\frac{\pi}{\ln (2)} \frac{V}{I} \mathrm{DMSO}$,

where $R_{\mathrm{S}}$ indicates the surface resistance, $V$ represents voltage and $I$ shows the current passed through the probes.

\section{Cell viability assay}

3-(4,5-Dimethylthiazol-2-yl)-2,5-diphenyltetrazolium bromide (MTT) assay was used to determine the viability of PC-12 cells seeded on the PCL/CNF scaffolds after 1 and 3 days of cell seeding. Scaffolds were placed in each well of 96-well plates and $1 \times 10^{4}$ of PC-12 cells were seeded onto each sample. The culture medium on the samples was replaced with $100 \mu \mathrm{L}$ of $0.5 \mathrm{mg} / \mathrm{mL}$ MTT solution and incubated for $4 \mathrm{~h}$. After this time, the MTT solution was aspirated and $100 \mu \mathrm{L}$ of dimethyl sulfoxide (DMSO) solution was added to dissolve the formazan crystals and kept at dark on a rotary shaker for $10 \mathrm{~min}$. The solution was transferred to new well and the absorbance values of the samples were read at $570 \mathrm{~nm}$.

\section{Cell adhesion studies}

A number of $7 \times 10^{3}$ PC- 12 cells were cultured on the electrospun scaffolds for 3 days. After this time period, the samples were washed with PBS and then fixed in 2.5\% 
glutaraldehyde solution at room temperature for $2 \mathrm{~h}$. The cell-scaffold constructs were then dehydrated using graded concentrations of ethanol in distilled water. The samples were freeze-dried and after sputter coating imaged under the SEM.

\section{In vivo studies}

\section{Sciatic nerve defect}

Male Wistar rats (3 months old, weighing 250-270 g) were purchased from Pasteur Institute (Tehran, Iran). Animal experiments were approved by the Ethics Committee of Tehran University of Medical Sciences and were carried out in accordance with the University guidelines. The animals were divided into 5 groups: (1) positive control (rats without sciatic nerve injury); (2) negative control (with sciatic nerve injury but without treatment); (3) autograft; (4) PCL/CNF group; (5) PCL group. To induce anesthesia, a mixture of ketamine 5\% and xylazine 2\% (70 mg ketamine and $6 \mathrm{mg}$ xylazine/1000 $\mathrm{g}$ body weight of animals) was administered by intraperitoneal injection. The right thigh of the rats was then shaved and disinfected by the povidone-iodine solution. A skin incision was made and the sciatic nerve was exposed. The sciatic nerve was severed in the middle and a 10-mm-long segment was resected. Proximal and distal nerve stumps were inserted into the NGCs leaving a 10-mm gap between two ends and the nerve tissue was secured in place by a 6-0 nylon suture. In the autograft group resected

Gastrocnemius muscle wet weight loss $(\%)=\left(1-\frac{\text { Wet weight of the muscle on the injured side }}{\text { Wet weight of the muscle on the uninjured side }}\right) \times 100$.
After 12 weeks of surgery, rats were killed and the pos-
terior gastrocnemius muscles at both operated and no-
operated limbs were harvested and immediately weighed
for the calculation of weight loss percentage by using the
following equation:

After 12 weeks of surgery, rats were killed and the pos-
terior gastrocnemius muscles at both operated and no-
operated limbs were harvested and immediately weighed
for the calculation of weight loss percentage by using the
following equation:

After 12 weeks of surgery, rats were killed and the pos-
terior gastrocnemius muscles at both operated and no-
operated limbs were harvested and immediately weighed
for the calculation of weight loss percentage by using the
following equation:

After 12 weeks of surgery, rats were killed and the pos-
terior gastrocnemius muscles at both operated and no-
operated limbs were harvested and immediately weighed
for the calculation of weight loss percentage by using the
following equation:

After 12 weeks of surgery, rats were killed and the pos-
terior gastrocnemius muscles at both operated and no-
operated limbs were harvested and immediately weighed
for the calculation of weight loss percentage by using the
following equation:

\section{Functional assessment of sensory recovery (hot plate test)}

The rat's sensory function recovery was investigated at 12 weeks post-surgery. Animals were placed on a hot plate $\left(56{ }^{\circ} \mathrm{C}\right)$ and the cut-off time was set at $12 \mathrm{~s}$. The time passed on the hot plate until the onset of rat's reaction (by licking the paws or jumping) was recorded as hot plate latency time (HPLT).

\section{Nerve conduction test}

Twelve weeks after surgery, the animals were anesthetized by intraperitoneal injection of ketamine $70 \mathrm{mg} / x y l a z i n e$ $6 \mathrm{mg} / \mathrm{kg}$ body weight) and the operated limb's sciatic nerve was exposed and stimulated just before the injury site with an electric stimulus (3-5 mA) using a needle electrode. Cap electrodes were placed on the gastrocnemius muscle (filtering frequency of $10 \mathrm{~Hz}$ to $10 \mathrm{kHz}$, the sensitivity of $2 \mathrm{mV} /$ division and sweep speed of $1 \mathrm{~ms} /$ division) and the compound muscle action potential (CMAP) amplitude was measured using an electromyographic recorder.

\section{Gastrocnemius muscle wet weight loss}

nerve was reversed and sutured back to the proximal and distal nerve ends.

\section{Walking-foot-print analysis}

Sciatic functional index (SFI) was investigated at 4th, 8th, and 12th week post-surgery. An acrylic corridor with the size of $43 \mathrm{~cm}$ length, $8.70 \mathrm{~cm}$ width and $5.50 \mathrm{~cm}$ height ended to a darkened goal box was prepared and its floor was covered by white papers. At specific time points, rat's hind paws were dipped into ink and allowed to walk through the corridor, and their foot prints were recorded on the papers. The SFI values for each group were calculated according to our previous study (Farzamfar et al. 2018). SFI $=0$ indicates the normal function, while the SFI $=-100$ represented the complete loss of function.

\section{Histopathological examination}

At the end of 12th-week post-surgery, the animals were killed with an overdose of ketamine $(150 \mathrm{mg} / \mathrm{kg}$ ketamine, $20 \mathrm{mg} / \mathrm{kg}$ xylazine) and their sciatic nerve and gastrocnemius muscle were harvested and fixed in a $10 \%$ buffered formalin. After processing and embedding in paraffin, they were sectioned and stained with hematoxylin-eosin (H\&E). The prepared samples were imaged under a light microscope (Carl Zeiss, Thornwood) with a digital camera.

\section{Statistical analysis}

The results were statistically analyzed by GraphPad prism (version 5) using one-way ANOVA and the data were 
expressed as mean \pm standard deviation (SD). In all evaluations, $P<0.05$ was considered as statistically significant.

\section{Results}

\section{Morphology of the scaffolds}

The SEM image of PCL/collagen nanofibers (Fig. 2a) illustrated that the fibers had a uniform and smooth morphology with random distribution. The fibrils' average diameter was measured using Image $\mathbf{J}$ (National Institutes of Health, Bethesda, USA) by selecting a total of 30 random points per image. Statistical analysis showed that the fibers had an average diameter of $1234.48 \pm 57.3 \mathrm{~nm}$. Electrospun scaffolds have remarkable effects in terms of cellular behavior such as cell orientation and differentiation. Neural cells migrate and deposit ECM proteins on nanofibrous matrices which have been shown to be beneficial for nerve repair (Cao et al. 2009; Nisbet et al. 2009; Yang et al. 2005). Figure 2b, c depicts the porous microstructure of PCL/CNF substrates. The obtained scaffolds had pore size around $100 \mu \mathrm{m}$ with suitable interconnectivity of the pores.

\section{Porosity measurement}

In order to increase the porosity of the NGCs, they were fabricated using the TIPS method. The high porosity facilitates metabolites diffusion into the NGCs which can provide a permissive environment for nerve repair following damage (de Ruiter et al. 2009). As shown in Table 1, incorporation of CNFs had a negligible effect on average porosity percentage of PCL scaffolds $(88.5 \pm 5.8 \%$ vs. $82.9 \pm 3.7 \%$ for PCL and $\mathrm{PCL} / \mathrm{CNF}$ scaffolds, respectively). It is accepted that porosity above $80 \%$ is ideal for tissue-engineered scaffolds (Salehi
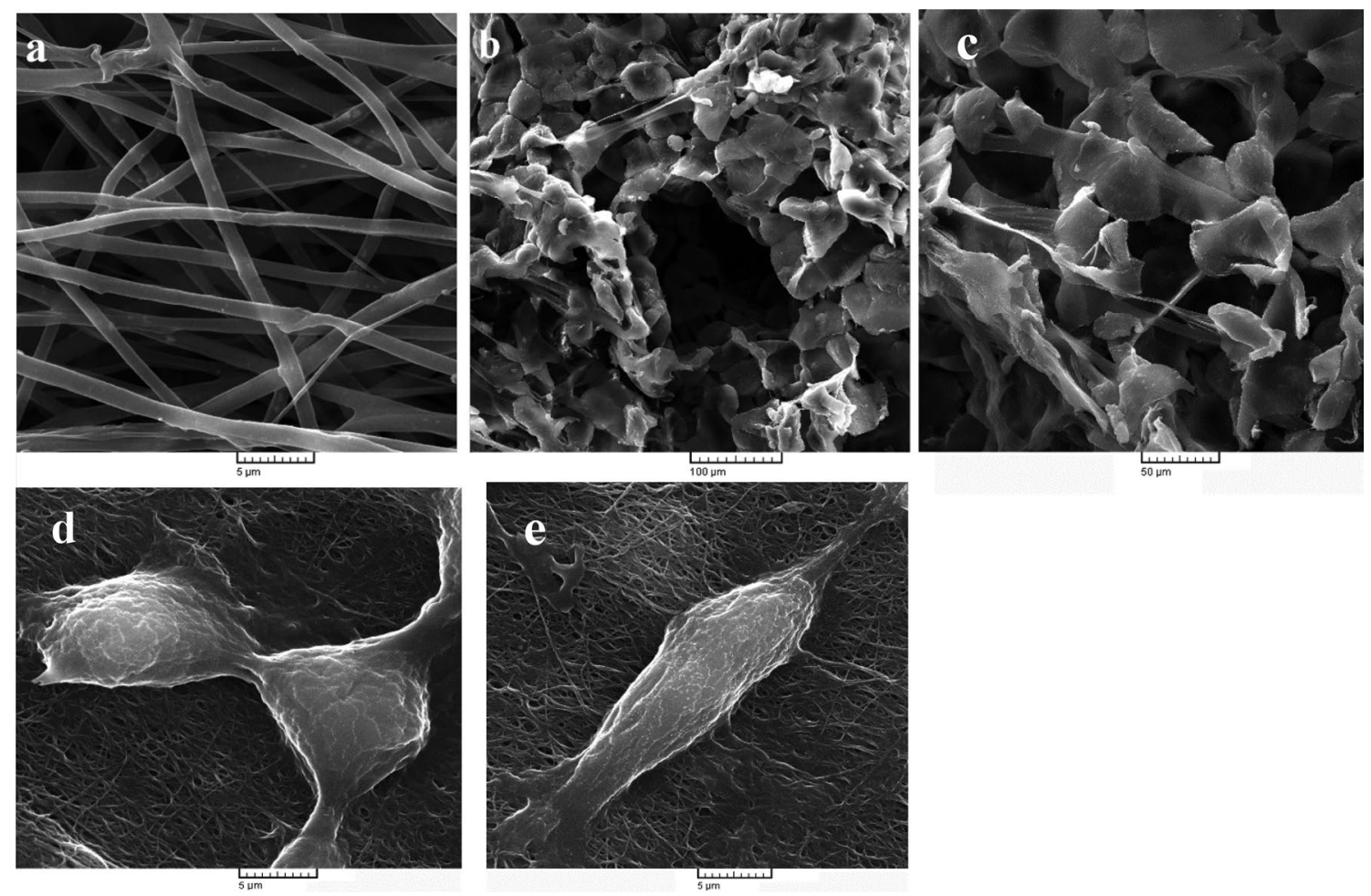

Fig. 2 a Representative SEM images of the PCL/collagen electrospun matrices; b, c SEM images of freeze-dried PCL/CNF composite scaffolds, and $\mathbf{e}, \mathbf{d}$ SEM images of PC-12 cells cultured on electrospun PCL/collagen nanofibrous scaffolds

Table 1 Characterization of the scaffolds. Values represent the mean $\pm \mathrm{SD}, n=3, * P<0.05$, $* * P<0.01$ and $* * * P<0.005$

\begin{tabular}{|c|c|c|c|c|}
\hline Samples & Electrical resistance $(\Omega)$ & Porosity (\%) & $\begin{array}{l}\text { Weight loss after } \\
30 \text { days }(\%)\end{array}$ & $\begin{array}{l}\text { Weight loss } \\
\text { after } 60 \text { days } \\
(\%)\end{array}$ \\
\hline PCL & $4.3 \times 10^{9} \pm .34 \times 10^{9}$ & $88.5 \pm 5.8$ & $2.17 \pm .54$ & $3.32 \pm .41$ \\
\hline PCL/CNF & $* * * 8.7 \times 10^{4} \pm 1.2 \times 10^{4}$ & $82.9 \pm 3.7$ & $1.84 \pm .37$ & $3.58 \pm .39$ \\
\hline
\end{tabular}


et al. 2018). Therefore, the fabrication technique utilized in this study could produce constructs with suitable porosity.

\section{Degradation rate measurement}

Ideal NGCs should be biodegradable to obviate the need for extra surgery for conduit removal after regeneration (de Ruiter et al. 2009). The results of in vitro degradation measurement (Table 1) showed that the PCL and PCL/ CNF scaffolds had a minimal weight loss percentage during 60 days. The average weight loss percentage between PCL and PCL/CNF groups was not statistically significant at both time intervals. This value for PCL/CNF scaffolds was $1.84 \pm 0.37 \%$ and $3.58 \pm 0.39 \%$ at 30th and 60th day, respectively. While PCL only scaffolds could degrade about $2.17 \pm 0.54 \%$ and $3.32 \pm 0.41 \%$ at 30th and 60th day, respectively. The ideal NGC should remain intact during the regeneration time and then should degrade gradually. High degradation rate may lead to swelling and blockade of the tunnel before the re-innervation of distal nerve stump (Muheremu and Ao 2015).

\section{Cell viability studies}

MTT assay was performed to evaluate the viability and proliferation of PC-12 cells on each scaffold. As shown in Fig. 3, at day 1 the activity of PC-12 cells grown on PCL and PCL/CNF scaffolds was lower than control group. However, the difference was not statistically significant. Cells seeded on PCL/CNF substrates demonstrated a significantly higher rate of cell proliferation compared to PCL only scaffolds at day 3 . Comparing the absorbance values of the control, PCL, and PCL/CNF groups showed that PCL and PCL/CNF scaffolds had no significant toxicity toward seeded cells. This is in accordance with previous studies which have reported proper cytocompatibility of

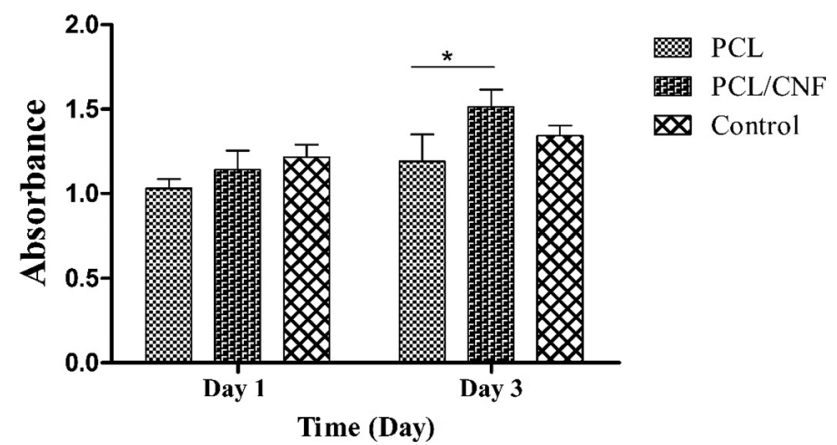

Fig. 3 Histogram comparing the viability of PC-12 cells cultured on the PCL and PCL/CNF scaffolds obtained by MTT assay 1 and 3 days after cell seeding. Values represent the mean $\pm \mathrm{SD}, n=3$, $* P<0.05, * * P<0.01$ and $* * * P<0.005$
CNF and PCL (Mirzaei et al. 2016; Naseri-Nosar et al. 2017).

\section{Cell attachment studies}

At the initial stage of cell growth on tissue engineering products, cell attachment which is highly dependent on surface chemistry governs tissue engineering progress (Dong et al. 2015). Scanning electron microscopy was utilized to assess the attachment of PC-12 cells on electrospun PCL/collagen scaffolds (Fig. 2d, e). From these figures, it is possible to observe cells grown on nanofibrous substrate which is well attached and spread out. It is also observable that cells have secreted ECM molecules.

\section{Electroconductivity measurement}

Electroactivity study revealed that incorporation of $\mathrm{CNF}$ into PCL matrix significantly reduced its electrical resistance from $4.3 \times 10^{9} \pm 0.34 \times 10^{9} \Omega$ to $8.7 \times 10^{4} \pm 1.2 \times 10^{4} \Omega$. The homogenously dispersed CNFs form percolated pathway required for an efficient electrical conductivity. The pathway helps the charge transports by hopping from one conductive site to the next and cause the composite to be electrically conductive (Salehi et al. 2018). Electrically conductive conduits can mimic the inherently conductive nature of the nerve tissues and therefore promote the regeneration of peripheral nerve injuries (Anderson et al. 2015).

\section{In vivo regenerative potential of NGCs}

\section{Sciatic function index (SFI)}

In animal models of sciatic nerve regeneration, SFI is widely used to evaluate the extent of motor function recovery (Lee et al. 2013). Figure 4 illustrates the measured SFI values for all groups. As shown the SFI for all

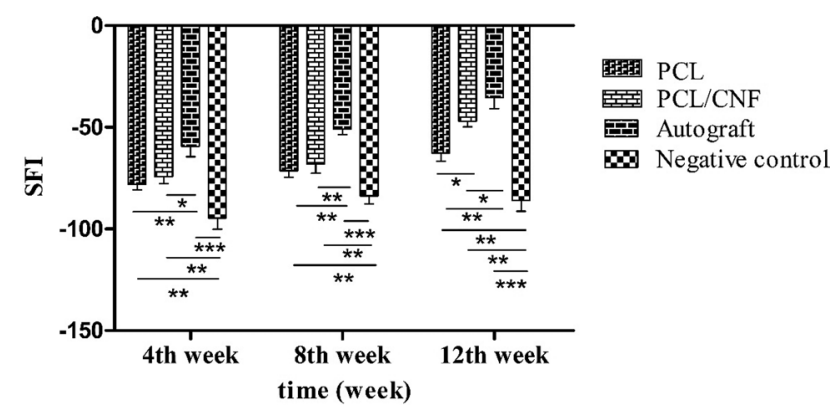

Fig. 4 Histogram comparing sciatic functional index study results of different groups 4,8 , and 12 weeks post-surgery. Values represent the mean $\pm \mathrm{SD}, n=3, * P<0.05, * * P<0.01$ and $* * * P<0.005$ 
groups had an increasing trend during the study period. Autograft group had significantly greater SFI compared to other groups at all time intervals $(p$ value $<0.05$ ) with the values of $-59.33 \pm 5.13,-51.32 \pm 2.64$, and $-35.66 \pm 3.21$ at the end of 4th, 8th, and 12th-week postsurgery, respectively. PCL/CNF group had the SFI results of $-74.33 \pm 3.19$ at the end of 4 th week. Its SFI reached the values of $-68 \pm 4.52$ and $-45.66 \pm 3.05$ at the end of 8 th and 12th week, respectively. The SFI values for PCL group were $-78.27 \pm 2.64,-71.33 \pm 4.21$, and $-62.66 \pm 4.01$ after 4,8 , and 12 weeks, respectively. PCL group had significantly lower SFI values compared to PCL/CNF at 12thweek post-surgery. SFI for the negative control group was close to -100 at all time intervals, implying complete neuromuscular degeneration.

\section{Hot plate latency time analysis}

The rats were monitored for their sensitivity to pain by hot plate latency test which reflects the degree of sensory function recovery. As shown in Fig. 5, the autograft group demonstrated a significantly ( $p$ value $<0.05$ ) better sensory recovery among all groups. However, the normal value of $4 \mathrm{~s}$ was not obtained (Hu et al. 1997). Although the animals in the PCL/CNF had a lower hot plate latency time at the end of the 12th-week post-surgery compared with PCL group, the difference was not statistically significant.

\section{Electrophysiological studies}

Compound muscle action potential (CMAP) indirectly indicates the motor nerves regeneration (Salehi et al. 2018). Measurements of this value through electrophysiological studies (Fig. 6) showed that severed nerves bridged with autograft nerve had the highest CMAP amplitude. These values for nerves implanted with PCL/CNF scaffolds were found to be significantly higher than that of the
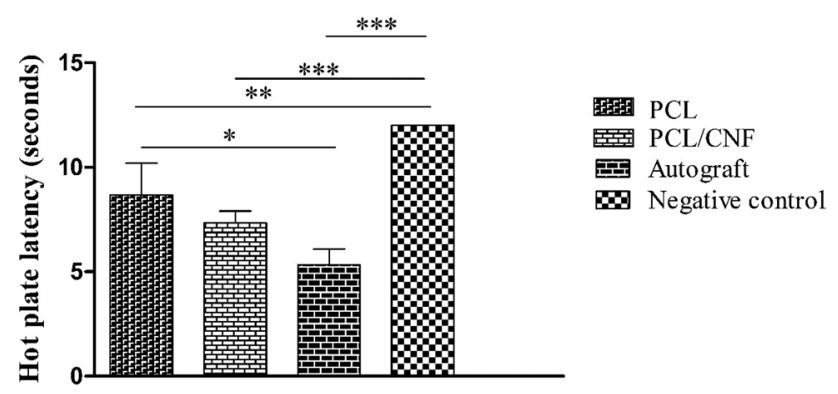

Fig. 5 Hot plate latency time measurement results 12 weeks postsurgery. Values represent the mean $\pm \mathrm{SD}, n=3, * P<0.05$, $* * P<0.01$ and $* * * P<0.005$

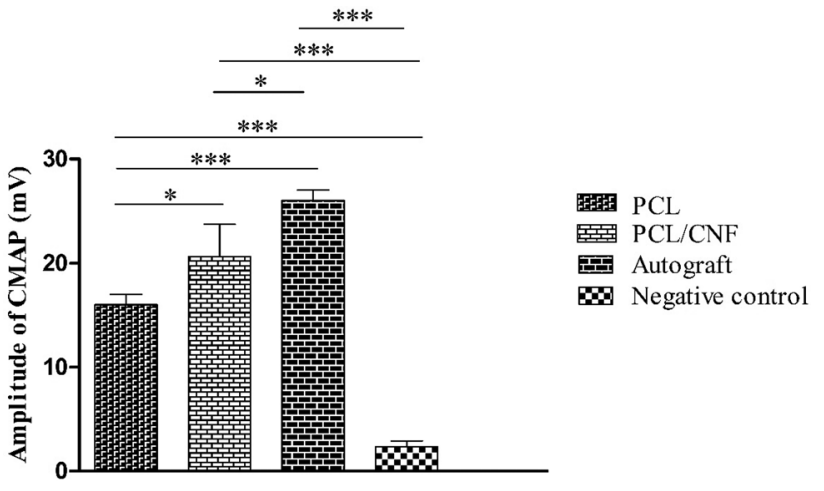

Fig. 6 Histogram comparing the amplitudes of compound muscle action potential 12 weeks after surgery. Values demonstrate the mean $\pm \mathrm{SD}, n=3, * P<0.05, * * P<0.01$ and $* * * P<0.005$

rats implanted with PCL conduits indicating higher myelin deposition in PCL/CNF group.

\section{Gastrocnemius muscle wet weight loss}

Sciatic nerve injury results in atrophy and weight loss of gastrocnemius muscle. The wet weight loss of the gastrocnemius muscle can indirectly reflect the efficacy of muscle re-innervation (Farzamfar et al. 2018). Figure 7 illustrates that the PCL/CNF group had a lower wet weight loss percentage of this muscle compared to PCL group. However, the difference was not statistically significant $(13.66 \pm 1.52 \%$ vs. $16.33 \pm 2.08, p$ value $<0.05$ ). Table 2 summarizes the results of functional analysis tests.

\section{Histopathological assessments}

After 12 weeks the animals were humanely killed and their sciatic nerve and gastrocnemius muscle tissues were removed for histopathological analysis. Then the gastrocnemius muscle was harvested and kept in $10 \%$ neutral buffered

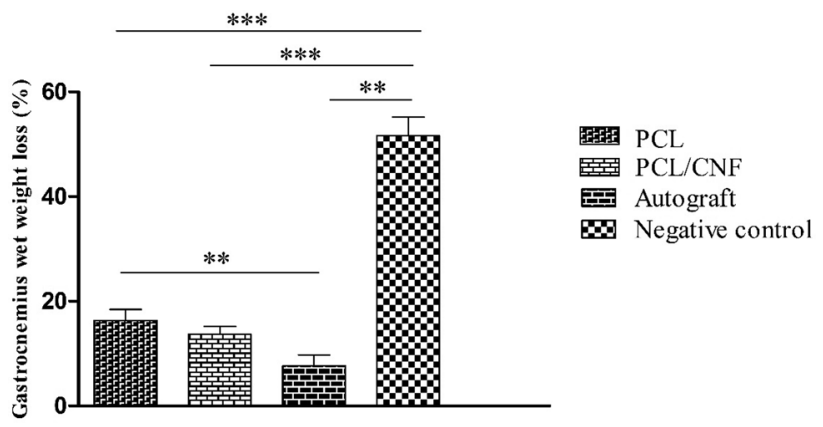

Fig. 7 Gastrocnemius muscle wet weight loss percentages of different groups after 12 weeks of surgery. Values represent the mean $\pm S D$, $n=3, * P<0.05, * * P<0.01$ and $* * * P<0.005$ 
Table 2 Summary of functional analysis results

\begin{tabular}{llllc}
\hline & $\begin{array}{l}\text { Sciatic function } \\
\text { index (12th week) }\end{array}$ & Hot plate latency time & $\begin{array}{l}\text { Compound muscle } \\
\text { action potential }\end{array}$ & $\begin{array}{l}\text { Gastrocnemius mus- } \\
\text { cle wet weight loss }\end{array}$ \\
\hline PCL & $-62.66 \pm 4.01$ & $8.66 \pm 1.52$ & $16.78 \pm 1.94$ & $16.33 \pm 2.08$ \\
PCL/CNF & $-45.66 \pm 3.05$ & $7.33 \pm .57$ & $20.66 \pm 3.05$ & $13.66 \pm 1.52$ \\
Autograft & $-35.66 \pm 3.21$ & $5.33 \pm .76$ & $26.45 \pm 2.54$ & $8.42 \pm 2.41$ \\
Negative control & $-86.00 \pm 5.29$ & 12 & $2.33 \pm .57$ & $51.73 \pm 3.60$ \\
\hline
\end{tabular}

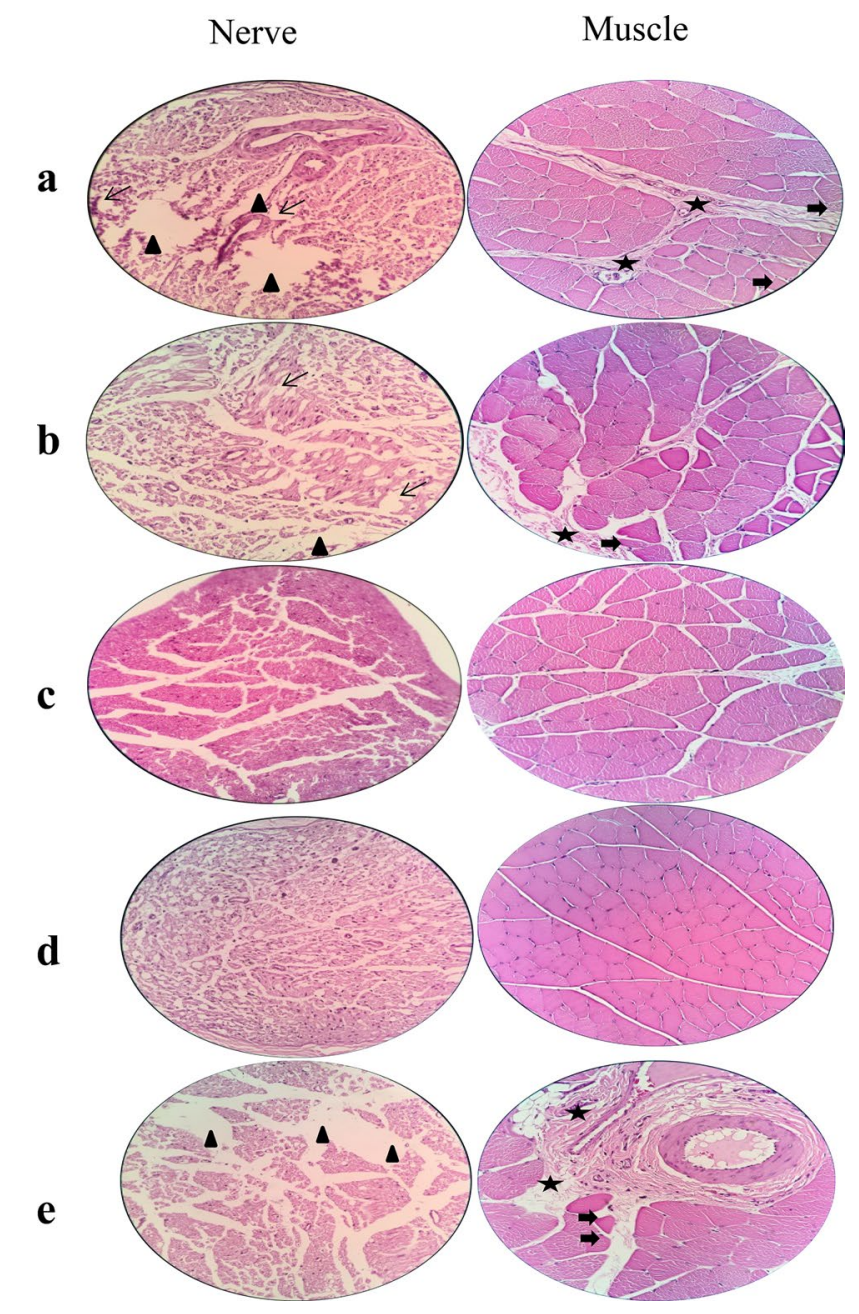

Fig. 8 Histopathological images of the sciatic nerve and gastrocnemius muscle $(\times 400)$ cross-sections stained by hematoxylin-eosin $(\mathrm{H} \& \mathrm{E})$ at the end of 12 th-week post-surgery. a PCL conduit, b PCL/ CNF conduit, $\mathbf{c}$ autograft, $\mathbf{d}$ positive control, e negative control. Narrow arrows: focal epineurium loss, asterisks: collagen hyperplasia, arrowheads: vacuolation, thickened arrows: atrophied muscle fiber

formalin (NBF, PH. 7.26) for 48 h. No signs of hematoma or infection were seen at the surgery site, indicating good tissue compatibility of the conduits which is in accordance with the MTT study. Figure 8 illustrates the histopathological examination of the sciatic nerves and gastrocnemius muscle of all groups. Microscopical analysis of the nerves in the positive control group demonstrated well-arranged myelinated nerves with no sign of histopathological alterations. In the negative control group, the nerve fibers were seriously damaged that was evidenced by fibrosis, swollen or degenerated nerve fibers accompanied by edema and high level of vacuolation. The histopathological evaluation of the PCL conduit group exhibited a various degree of vacuolation and mild edema. In the PCL/CNF conduit group, the nerve fibers were well-arranged and fibrosis or inflammatory cell infiltration were not seen in this sample. Histopathological study of the gastrocnemius muscle showed that in the positive control group, muscle fibers were intact indicating no pathological changes. On the other hand, the muscle fibers in the negative control group were contracted and degenerated. Furthermore, the myocytes striation was absent, and fibrosis was significantly increased between myocytes and the whole structure was totally damaged. Histopathological findings in the PCL/CNF conduit group demonstrated more like the autograft group. However, some degrees of fibrosis and muscular atrophy were seen. Muscular atrophy and various degrees of fibrosis and muscular shrinkage were observed in the PCL conduit group.

\section{Discussion}

In order to achieve a satisfactory motor and sensory recovery of an injured peripheral nerve, strategies seek to develop a substrate which exploits several cues to modulate neural cells behavior, whether it be to aid regenerating axons to reconnect with nerve distal stump or to encourage growth factors production and axonal sprouting (Gu et al. 2014). Electrical cues delivered through a conductive scaffold play a determining role in peripheral nerve repair following injury (Evans 2001). Several synthetic and/or natural materials have been tested for their applicability in neural tissue engineering (Daly et al. 2011). Among various candidates, CNFs possess unique mechanical and electrical properties that present new opportunities in the field of peripheral nerve tissue regeneration (Ku et al. 2013). In the current study, we examined the healing potential of CNF in the PCL matrix by comparing the regenerative capability of CNF containing and CNF free nerve guides in animal experiments. Overall, functional analysis and histopathological examinations showed that PCL/CNF nerve 
conduit enhanced nerve regeneration and mitigated muscle atrophy in a higher extent to what was achieved with CNF free NGCs. The higher healing activity of PCL/CNF conduits can be attributed to the inherent electrical conductivity of CNF. We suppose that higher regenerative performance of $\mathrm{CNF}$ containing conduits can be due to the fact that CNFs released from the scaffold changed the microenvironment in the NGCs lumen in a manner that was favorable for nerve regeneration. CNFs may transmit self-produced electrical cues between cells and the two ends of the severed sciatic nerve and improve cellular propagation and nerve regeneration (Ghasemi-Mobarakeh et al. 2011). This improved function of nerve tissues could be due to the better ultra-structural organization in the presence of electric fields. It is likely that neurons' surface receptors are rearranged in response to the presence of the field and signaling through voltage-gated calcium channels trigger ubiquitous second messengers such as cAMP and alter gene expression profile affecting survival and growth (Lanza et al. 2011). Previous studies have confirmed a positive interaction between $\mathrm{CNF}$ and neural cells. For example, Stout et al. speculated that higher neural cells densities on PLGA/CNF composites may be as a result of enhanced vitronectin and laminin deposition on CNFs, which in turn will trigger cell attachment and proliferation (Stout et al. 2011). Mirzaei et al. could successfully differentiate human endometrial stem cells into neuronlike cells on random and aligned carbon nanofibers (Mirzaei et al. 2016). Furthermore, previous studies have confirmed the higher healing potential of conductive NGCs compared to nonconductive ones. In this regard, we previously reported that multi-walled carbon nanotubes could significantly improve the electrical activity of polylactic acid nerve guides. In vivo studies revealed that carbon nanotube-containing group resulted in a higher nerve regeneration compared to the same conduit but without carbon nanostructures (Salehi et al. 2018). Das et al. tested the efficacy of a silk-based gold-nanocomposite NGC for peripheral nerve tissue engineering. Conduction measurement showed a 25 times decrease in silk nanofibers resistance when gold nanoparticles were incorporated into the silk matrix. Animal experiments proved the superior healing potential of gold nanoparticle containing conduits (Das et al. 2015). At this time the molecular mechanism involved in CNF's role in nerve regeneration is not clear, but this may be due to the aforementioned altered microenvironment and/or electrical activity, requiring further studies. With consideration of in vitro and in vivo results presented in this study, PCL/CNF conduits can be applied to treat peripheral nerve defects in clinic; however, adequate follow-ups regarding potential longterm adverse effects of CNFs should be taken into account.

\section{Conclusion}

In the current study, electrically conductive PCL/CNF conduits supported successful nerve regeneration in critical size sciatic nerve defect in the rat model. Further investigations need to be done to monitor long-term possible adverse effects of CNF application to treat nerve defects in the clinic. This preliminary research suggests the potential applicability of CNF in peripheral nerve tissue engineering.

Acknowledgements This study was supported by Tehran University of Medical Sciences (Grant No. 96-11-453002).

Author contributions All authors contributed to the study conception and design. Material preparation, data collection and analysis were performed by SF, JA, MS, KM, JV, AA, SMT, and ZVM. The first draft of the manuscript was written by SF and all authors commented on previous versions of the manuscript. All authors read and approved the final manuscript.

Funding This study was supported by Tehran University of Medical Sciences (Grant No. 96-11-453002).

\section{Compliance with ethical standards}

Conflict of interest The authors declare that there is no conflict of interests with regard to this study.

Ethical approval All applicable international, national, and/or institutional guidelines for the care and use of animals were followed. This article does not contain any studies with human participants performed by any of the authors.

Open Access This article is distributed under the terms of the Creative Commons Attribution 4.0 International License (http://creativeco mmons.org/licenses/by/4.0/), which permits unrestricted use, distribution, and reproduction in any medium, provided you give appropriate credit to the original author(s) and the source, provide a link to the Creative Commons license, and indicate if changes were made.

\section{References}

Anderson M, Shelke NB, Manoukian OS, Yu X, McCullough LD, Kumbar SG (2015) Peripheral nerve regeneration strategies: electrically stimulating polymer based nerve growth conduits. Crit Rev Biomed Eng 43:131

Cao H, Liu T, Chew SY (2009) The application of nanofibrous scaffolds in neural tissue engineering. Adv Drug Deliv Rev 61:1055-1064

Daly W, Yao L, Zeugolis D, Windebank A, Pandit A (2011) A biomaterials approach to peripheral nerve regeneration: bridging the peripheral nerve gap and enhancing functional recovery. J R Soc Interface 9:202-221

Das S, Sharma M, Saharia D, Sarma KK, Sarma MG, Borthakur BB, Bora U (2015) In vivo studies of silk based gold nano-composite conduits for functional peripheral nerve regeneration. Biomaterials 62:66-75 
de Ruiter GC, Malessy MJ, Yaszemski MJ, Windebank AJ, Spinner RJ (2009) Designing ideal conduits for peripheral nerve repair. Neurosurg Focus 26:E5

Dong Z (2015) Electrospinning and characterization of composite membranes for biomedical applications. Ph.D. Thesis, University of Rochester

Dong W et al (2015) A dual role of graphene oxide sheet deposition on titanate nanowire scaffolds for osteo-implantation: mechanical hardener and surface activity regulator. Sci Rep 5:18266

Evans GR (2001) Peripheral nerve injury: a review and approach to tissue engineered constructs. Anat Record Off Publ Am Assoc Anat 263:396-404

Faroni A, Mobasseri SA, Kingham PJ, Reid AJ (2015) Peripheral nerve regeneration: experimental strategies and future perspectives. Adv Drug Deliv Rev 82:160-167

Farzamfar S et al (2018) Neural tissue regeneration by a gabapentinloaded cellulose acetate/gelatin wet-electrospun scaffold. Cellulose 25:1229-1238

Ghasemi-Mobarakeh L et al (2011) Application of conductive polymers, scaffolds and electrical stimulation for nerve tissue engineering. J Tissue Eng Regenerat Med 5:e17-e35

Glowacki J, Mizuno S (2008) Collagen scaffolds for tissue engineering. Biopolym Origin Res Biomol 89:338-344

Gu X, Ding F, Williams DF (2014) Neural tissue engineering options for peripheral nerve regeneration. Biomaterials 35:6143-6156

Habre SB, Bond G, Jing XL, Kostopoulos E, Wallace RD, Konofaos $P$ (2018) The surgical management of nerve gaps: present and future. Ann Plast Surg 80:252-261

Hu D, Hu R, Berde CB (1997) Neurologic evaluation of infant and adult rats before and after sciatic nerve blockade. Anesthesiol J Am Soc Anesthesiol 86:957-965

Keane TJ, Badylak SF (2014) Biomaterials for tissue engineering applications. Semin Pediatr Surg 23:112-118

$\mathrm{Ku}$ SH, Lee M, Park CB (2013) Carbon-based nanomaterials for tissue engineering. Adv Healthcare Mater 2:244-260

Lannutti J, Reneker D, Ma T, Tomasko D, Farson D (2007) Electrospinning for tissue engineering scaffolds. Mater Sci Eng C 27:504-509

Lanza R, Langer R, Vacanti JP (2011) Principles of tissue engineering. Academic Press, Cambridge

Lee B-K, Ju YM, Cho J-G, Jackson JD, Lee SJ, Atala A, Yoo JJ (2012) End-to-side neurorrhaphy using an electrospun PCL/collagen nerve conduit for complex peripheral motor nerve regeneration. Biomaterials 33:9027-9036

Lee J-Y, Giusti G, Wang H, Friedrich PF, Bishop AT, Shin AY (2013) Functional evaluation in the rat sciatic nerve defect model: a comparison of the sciatic functional index, ankle angles, and isometric tetanic force. Plast Reconstr Surg 132:1173-1180

Mirzaei E, Ai J, Ebrahimi-Barough S, Verdi J, Ghanbari H, FaridiMajidi R (2016) The differentiation of human endometrial stem cells into neuron-like cells on electrospun PAN-derived carbon nanofibers with random and aligned topographies. Mol Neurobiol 53:4798-4808

Muheremu A, Ao Q (2015) Past, present, and future of nerve conduits in the treatment of peripheral nerve injury. BioMed Res Int 2015:237507

Naseri-Nosar M, Farzamfar S, Sahrapeyma H, Ghorbani S, Bastami F, Vaez A, Salehi M (2017) Cerium oxide nanoparticle-containing poly ( $\varepsilon$-caprolactone)/gelatin electrospun film as a potential wound dressing material: in vitro and in vivo evaluation. Mater Sci Eng C 81:366-372

Nectow AR, Marra KG, Kaplan DL (2011) Biomaterials for the development of peripheral nerve guidance conduits. Tissue Eng Part B Rev 18:40-50

Nisbet D, Forsythe JS, Shen W, Finkelstein D, Horne MK (2009) A review of the cellular response on electrospun nanofibers for tissue engineering. J Biomater Appl 24:7-29

Prabhakaran MP, Venugopal J, Chan CK, Ramakrishna S (2008) Surface modified electrospun nanofibrous scaffolds for nerve tissue engineering. Nanotechnology 19:455102

Salehi M, Naseri Nosar M, Amani A, Azami M, Tavakol S, Ghanbari H (2015) Preparation of pure PLLA, pure chitosan, and PLLA/ chitosan blend porous tissue engineering scaffolds by thermally induced phase separation method and evaluation of the corresponding mechanical and biological properties. Int J Polym Mater Polym Biomater 64:675-682

Salehi M, Naseri-Nosar M, Ebrahimi-Barough S, Nourani M, Khojasteh A, Hamidieh AA et al (2018) Sciatic nerve regeneration by transplantation of Schwann cells via erythropoietin controlledreleasing polylactic acid/multiwalled carbon nanotubes/gelatin nanofibrils neural guidance conduit. J Biomed Mater Res Part B Appl Biomater 106:1463-1476

Sarker M, Naghieh S, McInnes AD, Schreyer DJ, Chen X (2018) Strategic design and fabrication of nerve guidance conduits for peripheral nerve regeneration. Biotechnol J 13(7): 1700635

Schnell E, Klinkhammer K, Balzer S, Brook G, Klee D, Dalton P, Mey J (2007) Guidance of glial cell migration and axonal growth on electrospun nanofibers of poly- $\varepsilon$-caprolactone and a collagen/ poly- $\varepsilon$-caprolactone blend. Biomaterials 28:3012-3025

Siemionow M, Brzezicki G (2009) Current techniques and concepts in peripheral nerve repair. Int Rev Neurobiol 87:141-172

Smith BT, Shum J, Wong M, Mikos AG, Young S (2015) Bone tissue engineering challenges in oral \& maxillofacial surgery. In: Bertassoni L, Coelho P (eds) Engineering mineralized and load bearing tissues. Springer, Cham, pp 57-78

Stout DA, Basu B, Webster TJ (2011) Poly (lactic-co-glycolic acid): carbon nanofiber composites for myocardial tissue engineering applications. Acta Biomater 7:3101-3112

Wang HB, Mullins ME, Cregg JM, Hurtado A, Oudega M, Trombley MT, Gilbert RJ (2008) Creation of highly aligned electrospun poly-L-lactic acid fibers for nerve regeneration applications. J Neural Eng 6:016001

Yang F, Murugan R, Wang S, Ramakrishna S (2005) Electrospinning of nano/micro scale poly (L-lactic acid) aligned fibers and their potential in neural tissue engineering. Biomaterials 26:2603-2610

$\mathrm{Yu} \mathrm{W}$ et al (2011) Sciatic nerve regeneration in rats by a promising electrospun collagen/poly ( $\varepsilon$-caprolactone) nerve conduit with tailored degradation rate. BMC Neurosci 12:68

Zheng W et al (2012) Endothelialization and patency of RGD-functionalized vascular grafts in a rabbit carotid artery model. Biomaterials 33:2880-2891

Publisher's Note Springer Nature remains neutral with regard to jurisdictional claims in published maps and institutional affiliations. 